\title{
Translational Highlights
}

(C) Springer Science+Business Media, LLC 2012

\begin{abstract}
s
The following abstracts from The Endocrine Society journals have been selected by the editors as being particularly relevant to readers interested in translational science.
\end{abstract}

\section{Disruption of the Chemokine-Like Receptor-1 (CMKLR1) Gene Is Associated with Reduced Adiposity and Glucose Intolerance}

Matthew C. Ernst, Ian D. Haidl, Luis A. Zúñiga, Helen J. Dranse, Jillian L. Rourke, Brian A. Zabel, Eugene C. Butcher, and Christopher J. Sinal

Adipose tissue secretes a variety of bioactive signaling molecules, termed adipokines, which regulate numerous biological functions including appetite, energy balance, glucose homeostasis, and inflammation. Chemerin is a novel adipokine that regulates adipocyte differentiation and metabolism by binding to and activating the $G$ protein-coupled receptor, chemokine like receptor-1 (CMKLR1). In the present study, we investigated the impact of CMKLR1 deficiency on adipose development, glucose homeostasis, and inflammation in vivo. Herein we report that regardless of diet (low or high fat), $\mathrm{CMKLR}^{-/-}$mice had lower food consumption, total body mass, and percent body fat compared with wildtype controls. CMKLR1 $1^{-/}$mice also exhibited decreased hepatic and white adipose tissue TNF $\alpha$ and IL-6 mRNA levels coincident with decreased hepatic dendritic cell infiltration, decreased adipose CD3 $+\mathrm{T}$ cells, and increased adipose natural killer cells. CMKLR $1^{-/}$mice were glucose intolerant compared with wild-type mice, and this was associated with decreased glucose stimulated insulin secretion as well as decreased skeletal muscle and white adipose tissue glucose uptake. Collectively these data provide compelling evidence that CMKLR1 influences adipose tissue development, inflammation, and glucose homeostasis and may contribute to the metabolic derangement characteristic of obesity and obesity-related diseases.

This article appears in Endocrinology, published December 20, 2011, 10.1210/en.2011-1490

Developmental Changes in GnRH Release in Response to Kisspeptin Agonist and Antagonist in Female Rhesus Monkeys (Macaca mulatta): Implication for the Mechanism of Puberty

Kathryn A. Guerriero, Kim L. Keen, Robert P. Millar, and Ei Terasawa

Kisspeptin (KP) and KP-1 receptor (KISS1R) have emerged as important upstream regulators in the control of puberty. However, how developmental changes in KPKISS1R contribute to the pubertal increase in GnRH release still remains elusive. In this study, we examined the effects of the KP agonist, human KP-10 (hKP-10), and the KP antagonist, peptide 234 , on in vivo GnRH release in prepubertal and pubertal ovarian-intact female rhesus monkeys using a microdialysis method. We found that direct infusion of hKP-10 into the medial basal hypothalamus and stalkmedian eminence region stimulated GnRH release in a doseresponsive manner, whereas infusion of peptide 234 suppressed GnRH release in both developmental stages. Because ovarian steroid feedback on GnRH release becomes prominent after the initiation of puberty in primates, we further examined whether ovarian steroids modify the GnRH response to hKP-10. Results demonstrate that the hKP-10-induced stimulation of GnRH release was eliminated by ovariectomy in pubertal, but not prepubertal, monkeys. Furthermore, replacement of estradiol into ovariectomized pubertal monkeys resulted in a partial recovery of the hKP-10-induced GnRH release. Collectively, these results suggest that a KISS1R-mediated mechanism, in 
addition to the pubertal increase in KP-54 release we previously reported, contributes to the pubertal increase in $\mathrm{GnRH}$ release and that there is a switch from an ovarian steroidindependent to -dependent mechanism in the response of GnRH to KP.

This article appears in Endocrinology, published December 13, 2011, 10.1210/en.2011-1565

\section{Galectin-9 and T Cell Immunoglobulin Mucin-3 Pathway} Is a Therapeutic Target for Type 1 Diabetes

Motoko Kanzaki, Jun Wada, Koichi Sugiyama, Atsuko Nakatsuka, Sanae Teshigawara, Kazutoshi Murakami, Kentaro Inoue, Takahiro Terami, Akihiro Katayama, Jun Eguchi, Hisaya Akiba, Hideo Yagita, and Hirofumi Makino

Galectin-9 (Gal-9), a ligand for T cell Ig mucin-3 (Tim-3), induces apoptosis in cluster of differentiation $4(\mathrm{CD} 4)^{+} \mathrm{Tim}-$ $3^{+} \mathrm{T}$ helper $1\left(\mathrm{~T}_{\mathrm{H}} 1\right)$ cells via the Gal-9-Tim-3 pathway and negatively regulates $\mathrm{T}_{\mathrm{H}} 1$ immunity. In turn, Gal-9 activates dendritic cells (DC) to produce TNF- $\alpha$, which promotes the $\mathrm{T}_{\mathrm{H}} 1$ response. We investigated the efficacy of Gal-9 against $\mathrm{T}_{\mathrm{H}} 1$-mediated autoimmune diabetes in NOD mice and compared with anti-Tim-3 monoclonal antibody (RMT3-23), which inhibited the binding between Tim-3-Ig and Gal-9 in a solid-phase binding assay. mRNA expression of Gal-9 was prominently induced by the treatment of interferon- $\gamma$ in MIN6 cells, and Gal-9 was also expressed in the pancreatic $\beta$-cells in NOD mice, suggesting Gal-9 may be released from pancreatic $\beta$-cells to terminate $\mathrm{T}_{\mathrm{H}} 1$-mediated inflammation. Long-term injection of Gal-9 exhibits preventive efficacy for development of diabetes in NOD mice, but RMT3-23 demonstrated further prominent therapeutic potential compared with Gal-9. Gal-9 induced apoptosis of $\mathrm{CD}^{+}{ }^{+} \mathrm{Tim}-3^{+} \mathrm{T}_{\mathrm{H}} 1$ cells at the concentration of $0.2 \mu \mathrm{M}$, whereas RMT3-23 failed to enhance the apoptosis of CD4 ${ }^{+} \mathrm{Tim}_{-} 3^{+} \mathrm{T}_{\mathrm{H}} 1$ cells. In contrast, Gal-9 induced TNF- $\alpha$ production in cultured DC in a dose-dependent manner; however, RMT3-23 inhibited Gal-9-induced TNF- $\alpha$ production in a dose-dependent manner. Although Gal-9 exhibited certain therapeutic potential against autoimmune diabetes by enhancing apoptosis of $\mathrm{CD} 4^{+}$Tim- $3^{+} \mathrm{T}_{\mathrm{H}} 1$ cells, RMT3-23 exhibited prominent therapeutic efficacy by suppressing the TNF- $\alpha$ production and activation of DC. Taken together, the inhibition of the Gal-9-Tim-3 pathway on DC, upstream of $\mathrm{T}_{\mathrm{H}} 1$ response, may be a new target for the treatment of type 1 diabetes.

This article appears in Endocrinology, published December 20, 2011, 10.1210/en.2011-1579

\section{Late Intervention with anti-BRAF ${ }^{\mathrm{V} 600 \mathrm{E}}$ Therapy Induces Tumor Regression in an Orthotopic Mouse Model of Human Anaplastic Thyroid Cancer}

Matthew A. Nehs, Carmelo Nucera, Peter M. Sadow, Richard A. Hodin, and Sareh Parangi
Human anaplastic thyroid cancer (ATC) is a lethal disease with an advanced clinical presentation and median survival of 3 months. The $\mathrm{BRAF}^{\mathrm{V} 600 \mathrm{E}}$ oncoprotein is a potent transforming factor that causes human thyroid cancer cell progression in vitro and in vivo; therefore, we sought to target this oncoprotein in a late intervention model of ATC in vivo. We used the human ATC cell line 8505c, which harbors the $\mathrm{BRAF}^{\mathrm{V} 600 \mathrm{E}}$ and $\mathrm{TP} 53^{\mathrm{R} 248 \mathrm{G}}$ mutations. Immunocompromised mice were randomized to receive the selective anti$\mathrm{BRAF}^{\mathrm{V} 600 \mathrm{E}}$ inhibitor, PLX4720, or vehicle by oral gavage $28 \mathrm{~d}$ after tumor implantation, $1 \mathrm{wk}$ before all animals typically die due to widespread metastatic lung disease and neck compressive symptoms in this model. Mice were euthanized weekly to evaluate tumor volume and metastases. Control mice showed progressive tumor growth and lung metastases by $35 \mathrm{~d}$ after tumor implantation. At that time, all control mice had large tumors, were cachectic, and were euthanized due to their tumor-related weight loss. PLX4720-treated mice, however, showed a significant decrease in tumor volume and lung metastases in addition to a reversal of tumor-related weight loss. Mouse survival was extended to $49 \mathrm{~d}$ in PLX4720-treated animals. PLX4720 treatment inhibited cell cycle progression from $28 \mathrm{dto} 49 \mathrm{~d}$ in vivo. PLX4720 induces striking tumor regression and reversal of cachexia in an in vivo model of advanced thyroid cancer that harbors the $\mathrm{BRAF}^{\mathrm{V} 600 \mathrm{E}}$ mutation.

This article appears in Endocrinology, published December 27, 2011, 10.1210/en.2011-1519

\section{Curcumin Down-Regulates Visfatin Expression and Inhibits Breast Cancer Cell Invasion}

Su-Ryun Kim, Hyun-Joo Park, Yun-Hee Bae, SoonCheol Ahn, Hee-Jun Wee, Il Yun, Hye-Ock Jang, MoonKyoung Bae, and Soo-Kyung Bae

Obesity is frequently associated with breast cancer. Such associations are possibly mediated by adipokines. Visfatin, an adipokine, has recently been shown to be related to the development and progression of breast cancer. Therefore, the down-regulation of visfatin may be a novel strategy for breast cancer therapy. Curcumin has anticancer activities by modulating multiple signaling pathways and genes. The purpose of this study was to investigate whether visfatin gene expression is affected by curcumin in human breast cancer cells and to characterize the functional role of visfatin in breast cancer. We found that the mRNA and protein levels of visfatin were down-regulated by curcumin in MDA-MB231, MDA-MB-468, and MCF-7 breast cancer cells, along with decreased activity of constitutive nuclear factor (NF)$\mathrm{kB}$. We confirmed the repressive effect of curcumin on visfatin transcription by performing a visfatin promoterdriven reporter assay and identified two putative NF- $\mathrm{kB}$ binding sites on visfatin promoter that are important for this effect. EMSA and chromatin immunoprecipitation analysis 
indicated the binding of p65 to the visfatin promoter, which was effectively blocked by curcumin. Enforced expression of p65 protein increased visfatin promoter activity, whereas blocking NF- $\mathrm{kB}$ signaling suppressed visfatin gene expression. Visfatin could enhance the invasion of MDA-MB-231 cells and also attenuate curcumin-induced inhibition of cell invasion; on the other hand, visfatin knockdown by small interfering RNA led to the reduction of cell invasion. Our data demonstrates, for the first time, that curcumin downregulates visfatin gene expression in human breast cancer cells by a mechanism that is, at least in part, NF- $\mathrm{KB}$ dependent and suggest that visfatin may contribute to breast cancer cell invasion and link obesity to breast cancer development and progression.

This article appears in Endocrinology, published December 20, 2011, 10.1210/en.2011-1413

\section{Contribution of Nutritional Deficit to the Pathogenesis of the Nonthyroidal Illness Syndrome in Critical Illness: A Rabbit Model Study}

Liese Mebis, An Eerdekens, Fabian Güiza, Leslie Princen, Sarah Derde, Yoo-Mee Vanwijngaerden, Ilse Vanhorebeek, Veerle M. Darras, Greet Van den Berghe, and Lies Langouche

Both starvation and critical illness are hallmarked by changes in circulating thyroid hormone parameters with typically low $\mathrm{T}_{3}$ concentrations in the absence of elevated TSH. This constellation is labeled nonthyroidal illness (NTI). Because critical illness is often accompanied by anorexia and a failing gastrointestinal tract, the NTI of critical illness may be confounded by nutrient deficiency. In an experimental study performed in a rabbit model, we investigated the impact of nutritional deficit on the NTI of sustained critical illness. Critically ill rabbits were randomly allocated to parenteral nutrition (moderate dose $270 \mathrm{kcal} / \mathrm{d}$ ) initiated on the day after injury and continued until d 7 of illness or to infusing a similar volume of dextrose $1.4 \%(14 \mathrm{kcal} / \mathrm{d})$. With early parenteral nutrition during illness, the decrease in serum $\mathrm{T}_{3}$ observed with fasting was reversed, whereas the fall in $\mathrm{T}_{4}$ was not significantly affected. The rise in $\mathrm{T}_{3}$ with parenteral nutrition paralleled an increase of liver and kidney type-1 and a decrease of liver and kidney type- 3 deiodinase activity and an increase in circulating and central leptin. Nuclear staining of constitutive androstane receptor and its downstream expression of sulfotransferases were reduced in fasting ill animals. TRH expression in the hypothalamus was not different in fasted and fed ill rabbits, although circulating TSH levels were higher with feeding. In conclusion, in this rabbit model of sustained critical illness, reduced circulating $\mathrm{T}_{3}$, but not $\mathrm{T}_{4}$, levels could be prevented by parenteral nutrition, which may be mediated by leptin and its actions on tissue deiodinase activity.
This article appears in Endocrinology, published December 13, 2011, 10.1210/en.2011-1411

GLP-1 Receptor Agonists and the Thyroid: C-Cell Effects in Mice Are Mediated via the GLP-1 Receptor and not Associated with RET Activation

Lars Wichmann Madsen, Jeffrey A. Knauf, Carsten Gotfredsen, Andrew Pilling, Ingrid Sjögren, Søren Andersen, Lene Andersen, Anne Sietske de Boer, Katia Manova, Afsar Barlas, Sushil Vundavalli, Niels C. Berg Nyborg, Lotte Bjerre Knudsen, and James A. Fagin

Liraglutide and exenatide are glucagon-like peptide receptor (GLP-1R) agonists used in the treatment of type 2 diabetes. Both molecules have been associated with the development of thyroid C-cell tumors after lifetime exposure in rodents. Previously, it has been reported that these tumors are preceded by increased plasma calcitonin and $\mathrm{C}$ cell hyperplasia. We can now document that the murine $\mathrm{C}$ cell effects are mediated via GLP-1R. Thus, $13 \mathrm{wk}$ of continuous exposure to GLP-1R agonists was associated with marked increases in plasma calcitonin and in the incidence of C-cell hyperplasia in wild-type mice. In contrast, similar effects were not seen in GLP-1R knockout mice. Human C-cell cancer is often caused by activating mutations in the rearranged-during-transfection (RET) protooncogene. We developed an immunohistochemical method to assess $R E T$ activation in tissues. Liraglutide dosing to mice was not found to activate $R E T$. Further evaluation of the signaling pathways demonstrated that liraglutide increased ribosomal S6, but not MAPK kinase, phosphorylation. These observations are consistent with effects of GLP-1R agonists on rodent $\mathrm{C}$ cells being mediated via $m T O R$ activation in a RET-and MAPK-independent manner.

This article appears in Endocrinology, published January 10, 2012,10.1210/en.2011-1864

\section{Blockade of Cannabinoid Receptor 1 Improves Insulin Resistance, Lipid Metabolism, and Diabetic Nephropathy in $\mathbf{d b} / \mathbf{d b}$ Mice}

D. H. Nam, M. H. Lee, J. E. Kim, H. K. Song, Y. S. Kang, J. E. Lee, H. W. Kim, J. J. Cha, Y. Y. Hyun, S. H. Kim, S. Y. Han, K. H. Han, J. Y. Han, and Dae Ryong Cha

The endocannabinoid system is important in the pathogenesis of obesity-related metabolic disorders. However, the effect of inhibiting the endocannabinoid system in type 2 diabetic nephropathy is unclear. Therefore, we examined the effect of the cannabinoid $(\mathrm{CB}) 1$ receptor antagonist, SR141716, on insulin resistance and diabetic nephropathy in $\mathrm{db} / \mathrm{db}$ mice. Six-week-old $\mathrm{db} / \mathrm{db}$ mice were treated with the CB1-specific antagonist SR141716 (10 mg/kg·d) for 3 months. Treatment with SR141716 significantly improved insulin resistance and lipid abnormalities. Concomitantly, CB1 antagonism improved cardiac functional and 
morphological abnormality, hepatic steatosis, and phenotypic changes of adipocytes into small differentiated forms, associated with increased adiponectin expression and decreased lipid hydroperoxide levels. CB1 receptor was overexpressed in diabetic kidneys, especially in podocytes. Treatment with the SR141716 markedly decreased urinary albumin excretion and mesangial expansion and suppressed profibrotic and proinflammatory cytokine synthesis. Furthermore, SR141716 improved renal lipid metabolism and decreased urinary 8-isoprostane levels, renal lipid hydroperoxide content, and renal lipid content. In cultured podocytes, high-glucose stimulation increased CB1 receptor expression, and SR141716 treatment abolished highglucose-induced up-regulation of collagen and PAI-1 synthesis. Additionally, knockdown of CB1 receptor expression by stealth small interfering RNA abolished high-glucoseinduced sterol-regulatory element-binding protein-1 expression in podocytes. These findings suggest that CB1 blockade improves insulin resistance and protect against renal injury through both metabolic and antifibrotic effects in type 2 diabetic nephropathy. Targeting CB1 blockade could therefore provide a new therapeutic target to prevent type 2 diabetic nephropathy.

This article appears in Endocrinology, published January 10, 2012, 10.1210/en.2011-1423

\section{Thyroid Hormone Receptors Control Developmental Maturation of the Middle Ear and the Size of the Ossicular Bones}

Emily A. Cordas, Lily Ng, Arturo Hernandez, Masahiro Kaneshige, Sheue-Yann Cheng, and Douglas Forrest

Thyroid hormone is critical for auditory development and has well-known actions in the inner ear. However, less is known of thyroid hormone functions in the middle ear, which contains the ossicles (malleus, incus, stapes) that relay mechanical sound vibrations from the outer ear to the inner ear. During the later stages of middle ear development, prior to the onset of hearing, middle ear cavitation occurs, involving replacement of mesenchyme in the middle ear cavity with air, whereas the immature cartilaginous ossicles attain appropriate size and ossify. Using in situ hybridization, we detected expression of Thra and Thrb genes encoding thyroid hormone receptors- $\alpha 1$ and $-\beta$ (TR $\alpha 1$ and TR $\beta$, respectively) in the immature ossicles, surrounding mesenchyme and tympanic membrane in the mouse. Thra ${ }^{+/ P V}$ mice that express a dominant-negative TR $\alpha 1$ protein exhibited deafness with elevated auditory thresholds and a range of middle ear abnormalities including chronic persistence of mesenchyme in the middle ear into adulthood, markedly enlarged ossicles, and delayed ossification of the ossicles. Congenitally hypothyroid $T s h r^{-/-}$mice and TRdeficient Thra1 ${ }^{-/} ; \mathrm{Thrb}^{-/}$mice displayed similar abnormalities. These findings demonstrate that middle ear maturation is TR dependent and suggest that the middle ear is a sensitive target for thyroid hormone in development.

This article appears in Endocrinology, 10.1210/ en.2011-1834

\section{Reduced Hippocampal Brain-Derived Neurotrophic Factor (BDNF) in Neonatal Rats after Prenatal Exposure to Propylthiouracil (PTU)}

Goutam Chakraborty, Alejandra Magagna-Poveda, Carolyn Parratt, Jason G. Umans, Neil J. MacLusky, and Helen E. Scharfman

Thyroid hormone is critical for central nervous system development. Fetal hypothyroidism leads to reduced cognitive performance in offspring as well as other effects on neural development in both humans and experimental animals. The nature of these impairments suggests that thyroid hormone may exert its effects via dysregulation of the neurotrophin brain-derived neurotrophic factor (BDNF), which is critical to normal development of the central nervous system and has been implicated in neurodevelopmental disorders. The only evidence of BDNF dysregulation in early development, however, comes from experimental models in which severe prenatal hypothyroidism occurred. By contrast, milder prenatal hypothyroidism has been shown to alter BDNF levels and BDNF-dependent functions only much later in life. We hypothesized that mild experimental prenatal hypothyroidism might lead to dysregulation of BDNF in the early postnatal period. BDNF levels were measured by ELISA at 3 or $7 \mathrm{~d}$ after birth in different regions of the brains of rats exposed to propylthiouracil (PTU) in the drinking water. The dose of PTU that was used induced mild maternal hypothyroidism. Pups, but not the parents, exhibited alterations in tissue BDNF levels. Hippocampal BDNF levels were reduced at both $\mathrm{d} 3$ and 7 , but no significant reductions were observed in either the cerebellum or brain stem. Unexpectedly, more males than females were born to PTU-treated dams, suggesting an effect of PTU on sex determination. These results support the hypothesis that reduced hippocampal BDNF levels during early development may contribute to the adverse neurodevelopmental effects of mild thyroid hormone insufficiency during pregnancy.

This article appears in Endocrinology, published 2011, 10.1210/en.2011-1437

\section{Uncovering Novel Reproductive Defects in Neurokinin B Receptor Null Mice: Closing the Gap Between Mice and Men}

Jasmine Y. Yang, Claudia S. Caligioni, Yee-Ming Chan, and Stephanie B. Seminara

Patients bearing mutations in TAC3 and TACR3 (which encode neurokinin $\mathrm{B}$ and its receptor, respectively) have sexual infantilism and infertility due to GnRH deficiency. 
In contrast, $\mathrm{Tacr} 3^{-/-}$mice have previously been reported to be fertile. Because of this apparent phenotypic discordance between mice and men bearing disabling mutations in Tacr3/TACR3, Tacr3 null mice were phenotyped with close attention to pubertal development, estrous cyclicity, and fertility. Tacr $3^{--}$mice demonstrated normal timing of preputial separation and day of first estrus, markers of sexual maturation. However, at postnatal d 60, TaCr $^{3^{--}}$males had significantly smaller testes and lower FSH levels than their wild-type littermates. Tacr $3^{-/}$females had lower uterine weights and abnormal estrous cyclicity. Approximately half of Tacr $^{-/-}$females had no detectable corpora lutea on ovarian histology at postnatal d 60. Despite this apparent ovulatory defect, all $\mathrm{Tacr}^{-/}$females achieved fertility when mated. However, Tacr $3^{-/}$females were subfertile, having both reduced numbers of litters and pups per litter. The subfertility of these animals was not due to a primary ovarian defect, because they demonstrated a robust response to exogenous gonadotropins. Thus, although capable of fertility, Tacr3-deficient mice have central reproductive defects. The remarkable ability of acyclic female Tacr3 null mice to achieve fertility is reminiscent of the reversal of hypogonadotropic hypogonadism seen in a high proportion of human patients bearing mutations in TACR3. Tacr 3 mice are a useful model to examine the mechanisms by which neurokinin B signaling modulates GnRH release.

This article appears in Endocrinology, published 2011, 10.1210/en.2011-1949

A SNP in Steroid Receptor Coactivator-1 Disrupts a GSK3 $\beta$ Phosphorylation Site and Is Associated with Altered Tamoxifen Response in Bone

R. J. Hartmaier, A. S. Richter, R. M. Gillihan, J. Z. Sallit, S. E. McGuire, J. Wang A. V. Lee, C. K. Osborne, B. W. O’Malley, P. H. Brown, J. Xu, T. C. Skaar, S. Philips, J. M. Rae, F. Azzouz, L. Li, J. Hayden, N. L. Henry, A. T. Nguyen, V. Stearns, D. F. Hayes, D. A. Flockhart,and S. Oesterreich

The coregulator steroid receptor coactivator (SRC)-1 increases transcriptional activity of the estrogen receptor (ER) in a number of tissues including bone. Mice deficient in SRC-1 are osteopenic and display skeletal resistance to estrogen treatment. SRC-1 is also known to modulate effects of selective ER modulators like tamoxifen. We hypothesized that single nucleotide polymorphisms (SNP) in SRC-1 may impact estrogen and/or tamoxifen action. Because the only nonsynonymous SNP in SRC-1 (rs1804645; P1272S) is located in an activation domain, it was examined for effects on estrogen and tamoxifen action. SRC-1 P1272S showed a decreased ability to coactivate ER compared with wild-type SRC-1 in multiple cell lines. Paradoxically, SRC-1 P1272S had an increased protein half-life. The Pro to Ser change disrupts a putative glycogen synthase 3 (GSK3) $\beta$ phosphorylation site that was confirmed by in vitro kinase assays.
Finally, knockdown of GSK3 $\beta$ increased SRC-1 protein levels, mimicking the loss of phosphorylation at P1272S. These findings are similar to the GSK $3 \beta$-mediated phospho-ubiquitin clock previously described for the related coregulator SRC-3. To assess the potential clinical significance of this SNP, we examined whether there was an association between SRC-1 P1272S and selective ER modulators response in bone. SRC-1 P1272S was associated with a decrease in hip and lumbar bone mineral density in women receiving tamoxifen treatment, supporting our in vitro findings for decreased ER coactivation. In summary, we have identified a functional genetic variant of SRC-1 with decreased activity, resulting, at least in part, from the loss of a GSK $3 \beta$ phosphorylation site, which was also associated with decreased bone mineral density in tamoxifen-treated women.

This article appears in Molecular Endocrinology, published December 15, 2011, 10.1210/me.2011-1032

The RANKL Distal Control Region Is Required for the Increase in RANKL Expression, But Not the Bone Loss, Associated with Hyperparathyroidism or Lactation in Adult Mice

Melda Onal, Carlo Galli, Qiang Fu, Jinhu Xiong, Robert S. Weinstein, Stavros C. Manolagas, and Charles A. O'Brien

Osteoclast-mediated bone resorption plays an essential role in calcium homeostasis and lactation. The cytokine receptor activator of nuclear factor $\mathrm{KB}$ ligand (RANKL) is one of a number of factors that controls the production, survival, and activity of osteoclasts. Calciotropic hormones, such as PTH, control RANKL transcription in part via an enhancer known as the distal control region (DCR), and mice lacking this enhancer have fewer osteoclasts under normal physiological conditions. Here, we have addressed the role of the DCR in situations in which activation of the PTH receptor is thought to stimulate bone resorption via elevation of RANKL expression. Dietary calcium deficiency stimulated RANKL expression in the bone of young (1 month old) wild-type, but not DCR knockout (KO), mice. Consistent with this, the cancellous bone loss and the increase in osteoclasts caused by dietary calcium deficiency were blunted in young KO mice. DCR deletion also prevented the increase in RANKL expression caused by dietary calcium deficiency in 6-month-old mice. However, the diet-induced bone loss was similar in wild-type and $\mathrm{KO}$ mice at this age. The increase in RANKL expression caused by lactation was also blunted in DCR KO mice, but lactationinduced bone loss was similar in both genotypes. These results demonstrate that, even though the DCR is required for the increase in RANKL expression associated with hyperparathyroidism or lactation, this increase is not required for the bone 
loss caused by these conditions in adult mice, suggesting that changes in other factors, such as osteoprotegerin or estrogen levels, play a dominant role.

This article appears in Molecular Endocrinology, published December 29, 2011, 10.1210/me.2011-1149

\section{Loss of FXR Protects against Diet-Induced Obesity and} Accelerates Liver Carcinogenesis in ob/ob Mice

Yanqiao Zhang, Xuemei Ge, Lydia A. Heemstra, WeiDong Chen, Jiesi Xu, Joseph L Smith, Huiyan Ma, Neda Kasim, Peter A. Edwards, and Colleen M. Novak

Farnesoid X receptor (FXR) is known to play important regulatory roles in bile acid, lipid, and carbohydrate metabolism. Aged ( $>12$ months old) $\mathrm{Fxr}^{-/-}$mice also develop spontaneous liver carcinomas. In this report, we utilized three mouse models to investigate the role of FXR deficiency in obesity. As compared to low-density lipoprotein receptor $(L d l r)$ knockout $\left(L d l r^{-/}\right)$mice, the $L d l r^{-/} F x r^{-/}$double knockout mice were highly resistant to diet-induced obesity, which was associated with increased expression of genes involved in energy metabolism in the skeletal muscle and brown adipose tissue. Such a striking effect of FXR deficiency on obesity on an $\mathrm{Ldll}^{-/-}$background led us to investigate whether FXR deficiency alone is sufficient to affect obesity. As compared to wild-type mice, $\mathrm{Fxr}^{-/-}$mice showed resistance to diet-induced weight gain. Interestingly, only female $\mathrm{Fxr}^{-/}$mice showed significant resistance to dietinduced obesity, which was accompanied by increased energy expenditure in these mice. Finally, we determined the effect of FXR deficiency on obesity in a genetically obese and diabetic mouse model. We generated $\mathrm{ob}^{-/} \mathrm{Fxr}^{-/-}$mice that were deficient in both Leptin and Fxr. On a chow diet, $o b^{-/} F x r^{-/}$mice gained less body weight and had reduced body fat mass as compared to $o b / o b$ mice. In addition, we observed liver carcinomas in $43 \%$ of young $(<11$ months old) $\mathrm{ob}^{-/} \mathrm{Fxr}^{-/-}$mice. Together, these data indicate that loss of FXR prevents diet-induced or genetic obesity and accelerates liver carcinogenesis under diabetic conditions.

This article appears in Molecular Endocrinology, 10.1210/me.2011-1157

\section{Intraovarian Thrombin and Activated Protein C Signaling System Regulates Steroidogenesis during the Periovulatory Period}

Yuan Cheng, Kazuhiro Kawamura, Masashi Deguchi, Seido Takae, Sabine M. Mulders, and Aaron J. W. Hsueh

In addition to its role in blood coagulation, thrombin directly stimulates protease-activated receptors (PAR) or interacts with thrombomodulin (THBD) to activate membrane-bound protein $\mathrm{C}$ which stimulates PAR1 and PAR4 receptors to promote downstream pleiotropic effects. Our DNA microarray, RT-PCR, and immunostaining analyses demonstrated ovarian expression of THBD, activated protein $\mathrm{C}$ (APC) receptor [endothelial protein $\mathrm{C}$ receptor (EPCR)], as well as PAR1 and PAR4 receptors in mice. After treatment of gonadotropin-primed immature mice with an ovulatory dose of human chorionic gonadotropin (hCG) (a LH surrogate), major increases in the expression of THBD, EPCR, PAR1, and PAR4 were detected in granulosa and cumulus cells of preovulatory follicles. Immunoassay analyses demonstrated sustained increases in ovarian prothrombin and APC levels after hCG stimulation. We obtained luteinizing granulosa cells from mice treated sequentially with equine CG and hCG. Treatment of these cells with thrombin or agonists for PAR1 or PAR4 decreased basal and forskolin-induced cAMP biosynthesis and suppressed hCG-stimulated progesterone production. In cultured preovulatory follicles, treatment with hirudin (a thrombin antagonist) and SCH79797 (a PAR1 antagonist) augmented hCG-stimulated progesterone biosynthesis, suggesting a suppressive role of endogenous thrombin in steroidogenesis. Furthermore, intrabursal injection with hirudin or SCH79797 led to ipsilateral increases in ovarian progesterone content. Our findings demonstrated increased ovarian expression of key components of the thrombin-APC-PAR1/4 signaling system after LH/hCG stimulation, and this signaling pathway may allow optimal luteinization of preovulatory follicles. In addition to assessing the role of thrombin and associated genes in progesterone production by the periovulatory ovary, these findings provide a model with which to study molecular mechanisms underlying thrombin-APC-PAR1/4 signaling.

This article appears in Molecular Endocrinology, published December 29, 2011, 10.1210/me.2011-1187

\section{Deletion of CaMKK2 from the Liver Lowers Blood Glucose and Improves Whole-Body Glucose Tolerance in the Mouse}

Kristin A. Anderson, Fumin Lin, Thomas J. Ribar, Robert D. Stevens, Michael J. Muehlbauer, Christopher B. Newgard, and Anthony R. Means

$\mathrm{Ca}^{2+} /$ calmodulin-dependent protein kinase kinase (CaMKK2) is a member of the $\mathrm{Ca}^{2+} / \mathrm{CaM}$-dependent protein kinase family that is expressed abundantly in brain. Previous work has revealed that CaMKK2 knockout (CaMKK2 KO) mice eat less due to a central nervous system -signaling defect and are protected from diet-induced obesity, glucose intolerance, and insulin resistance. However, here we show that pair feeding of wild-type mice to match food consumption of CAMKK2 mice slows weight gain but fails to protect from diet-induced glucose intolerance, suggesting that other alterations in CaMKK2 KO mice are responsible for their improved glucose metabolism. CaMKK2 is shown to be expressed in liver and acute, specific reduction of the kinase from the liver of high-fat diet-fed CaMKK2 $2^{\text {floxed }}$ mice results in lowered blood glucose and improved glucose 
tolerance. Primary hepatocytes isolated from CaMKK2 KO mice produce less glucose and have decreased mRNA encoding peroxisome proliferator-activated receptor $\gamma$ coactivator $1-\alpha$ and the gluconeogenic enzymes glucose6phosphatase and phosphoenolpyruvate carboxykinase, and these mRNA fail to respond specifically to the stimulatory effect of catecholamine in a cell-autonomous manner. The mechanism responsible for suppressed gene induction in CaMKK2 KO hepatocytes may involve diminished phosphorylation of histone deacetylase 5 , an event necessary in some contexts for derepression of the peroxisome proliferator-activated receptor $\{$ coactivator $1-\}$ promoter. Hepatocytes from CaMKK2 KO mice also show increased rates of de novo lipogenesis and fat oxidation. The changes in fat metabolism observed correlate with steatotic liver and altered acyl carnitine metabolomic profiles in CaMKK2 KO mice. Collectively, these results are consistent with suppressed catecholamine-induced induction of gluconeogenic gene expression in CaMKK2 $\mathrm{KO}$ mice that leads to improved whole-body glucose homeostasis despite the presence of increased hepatic fat content.

This article appears in Molecular Endocrinology, published 2011, 10.1210/me.2011-1299

\section{Differential Expression of the Androgen-Conjugating} UGT2B15 and UGT2B17 Enzymes in Prostate Tumor Cells during Cancer Progression

Sophie Pâquet, Ladan Fazli, Laurent Grosse, Mélanie Verreault, Bernard Têtu, Paul S. Rennie, Alain Bélanger, and Olivier Barbier

Context: Androgens play major roles in prostate cancer initiation and development. In prostate cells, the human uridine diphosphate-glucuronosyltransferase (UGT)2B15 and UGT2B17 enzymes inactivate androgens.

Objective: We investigated in vivo how UGT2B15 and UGT2B17 expressions are affected during prostate cancer development.

Design: We conducted an observational study of the UGT2B15 and UGT2B17 mRNA and protein levels.

Setting: The study was conducted at Laval University (Québec, Canada) and at the University of British Columbia (Vancouver, Canada).

Patients/Participants: Participants were from a cohort of prostate cancer patients from the Hôtel-Dieu de Québec hospital (Québec; mRNA analyses) and from the Vancouver Prostate Centre tissue bank (Vancouver; tissue microarray experiments).

Main Outcome Measures: UGT mRNA and protein levels were determined using real-time PCR and immunohistochemical analyses, respectively.

Results: Both UGT2B15 and UGT2B17 mRNA and protein levels were not significantly associated with Gleason score stratification. However, when protein levels were compared to benign prostatic hyperplasia, UGT2B17 was significantly more abundant in all Gleason-scored tumors. By contrast, UGT2B15 levels were significantly reduced in naive and castration-resistant tumors and undetectable in lymph node metastases. Finally, UGT2B17 proteins were 5-fold more abundant in metastases than in benign samples.

Conclusions: The current study reveals that UGT2B15 and UGT2B17 are differentially regulated during prostate cancer progression. Furthermore, this study also identifies the UGT2B15 gene as a negatively regulated target gene in castration-resistant prostate cancer and lymph node metastases.

This article appears in The Journal of Clinical Endocrinology \& Metabolism, published December 14, 2011, 10.1210/ jc.2011-2064

In Vitro Studies of Novel PRKAR1A Mutants that Extend the Predicted RI $\alpha$ Protein Sequence into the 3'-Untranslated Open Reading Frame: Proteasomal Degradation Leads to RI $\alpha$ Haploinsufficiency and Carney Complex

Yianna Patronas, Anelia Horvath, Elizabeth Greene, Kitman Tsang, Eirini Bimpaki, Michelle Haran, Maria Nesterova, and Constantine A. Stratakis

Background: Carney complex (CNC) is a multiple endocrine neoplasia syndrome due to inactivating mutations in the PRKARIA gene that codes for type $\mathrm{I} \alpha$ regulatory $(\mathrm{RI} \alpha)$ subunit of protein kinase A. Most PRKARIA mutations are subject to nonsense mRNA decay (NMD) and, thus, lead to haploinsufficiency.

Methods and Setting: Patient phenotyping for $\mathrm{CNC}$ features and DNA, RNA, protein, and transfection studies were carried out at a clinical research center.

Results: We describe four naturally occurring PRKAR1A mutations (1055del4, 1067del4ins5, 1076delTTins13, and 1142de14) in unrelated kindreds with $\mathrm{CNC}$ that are predicted to escape NMD because they are located in the last coding exon of the gene. The phenotype of CNC was not different from that in other patients with the condition, although the number of patients was small. Each of the mutations caused a frameshift that led to a new stop codon into the $3^{\prime}$ untranslated open reading frame, predicting an elongated protein that, however, was absent in patient-derived cells. After site-directed mutagenesis, in vitro transcription, and translation experiments the expected size mutant proteins were present in a cell-free system. When the mutant constructs were transfected in adrenal, testicular, and embryonic (HEK293) cells and despite the presence of the mutant mRNA, Western blot analysis indicated that there were no longer proteins. The subsequent application of proteasome inhibitors to cells transfected with the mutant constructs led to the detection of the aberrant proteins, although a compound that affects protein 
folding had no effect. The wild-type protein was also decreased in both patient-derived cells and/or tissues as well as in the in vitro systems used in this study.

Conclusions: This was the first demonstration of proteasomal degradation of $\mathrm{RI} \alpha$ protein variants leading to PRKAR1A haploinsufficiency and $\mathrm{CNC}$, adding protein surveillance to NMD in the cellular mechanisms overseeing $\mathrm{RI} \alpha$ synthesis. In agreement with the molecular data, CNC patients bearing PRKARIA defects that extend the open reading frame did not have a different phenotype, although this has to be confirmed in a larger number of patients.

This article appears in The Journal of Clinical Endocrinology \& Metabolism, published December 28, 2011, 10.1210/ jc. 2011-2220

\section{Acute Sleep Deprivation Enhances the Brain's Response to Hedonic Food Stimuli: An fMRI Study}

Christian Benedict, Samantha J. Brooks, Owen G. O’Daly, Markus S. Almèn, Arvid Morell, Karin Åberg, Malin Gingnell, Bernd Schultes, Manfred Hallschmid, Jan-Erik Broman, Elna-Marie Larsson, and Helgi B. Schiöth

Context: There is growing recognition that a large number of individuals living in Western society are chronically sleep deprived. Sleep deprivation is associated with an increase in food consumption and appetite. However, the brain regions that are most susceptible to sleep deprivationinduced changes when processing food stimuli are unknown.

Objective: Our objective was to examine brain activation after sleep and sleep deprivation in response to images of food.

Intervention: Twelve normal-weight male subjects were examined on two sessions in a counterbalanced fashion: after one night of total sleep deprivation and one night of sleep. On the morning after either total sleep deprivation or sleep, neural activation was measured by functional magnetic resonance imaging in a block design alternating between high-and lowcalorie food items. Hunger ratings and morning fasting plasma glucose concentrations were assessed before the scan, as were appetite ratings in response to food images after the scan.

Main Outcome Measures: Compared with sleep, total sleep deprivation was associated with an increased activation in the right anterior cingulate cortex in response to food images, independent of calorie content and prescan hunger ratings. Relative to the postsleep condition, in the total sleep deprivation condition, the activation in the anterior cingulate cortex evoked by foods correlated positively with postscan subjective appetite ratings. Self-reported hunger after the nocturnal vigil was enhanced, but importantly, no change in fasting plasma glucose concentration was found.

Conclusions: These results provide evidence that acute sleep loss enhances hedonic stimulus processing in the brain underlying the drive to consume food, independent of plasma glucose levels. These findings highlight a potentially important mechanism contributing to the growing levels of obesity in Western society.

This article appears in The Journal of Clinical Endocrinology \& Metabolism, 10.1210/jc.2011-2759

\section{EGF Receptor (ERBB1) Abundance in Adipose Tissue Is Reduced in Insulin-Resistant and Type 2 Diabetic Women}

Carlyle Rogers, Fatiha Moukdar, Marie A. McGee, Barbara Davis, Benjamin Buehrer, Kiefer W. Daniel, Sheila Collins, Hisham Barakat, and Jacques Robidoux

Context: Indications of adipose tissue dysfunction correlate with systemic insulin resistance and type 2 diabetes. It has been suggested that a defect in adipose tissue turnover may be involved in the development of these disorders. Whether this dysfunction causes or exacerbates systemic insulin resistance is not fully understood.

Objectives, Participants, and Measures: We tested whether the expression of members of the mitogenic ErbB family was reduced in adipose tissue of insulin-resistant individuals and whether ErbB1 and ErbB2 were involved in adipogenesis. Thirty-two women covering a wide range of body mass index values and insulin sensitivity participated in the crosssectional portion of this study. We also studied preadipocytes isolated from 12 insulin-sensitive individuals to evaluate the impact of ErbB1 or ErbB2 inhibition on adipogenesis in vitro. For this purpose, we measured phospho-ErbB1 and phosphoErbB2 levels using ELISA and the expression of peroxisome proliferator-activated receptor $\gamma(\operatorname{PPAR} \gamma)$ and PPAR $\gamma$ regulated genes by real-time PCR.

Results: Among the ErbB family members, only ErbB1 expression was correlated with insulin sensitivity. Additionally, ErbB1 levels correlated positively with PPAR $\gamma$ and several PPAR $\gamma$-regulated genes including acyl-coenzyme A synthetase long-chain family member 1 (ACSL1), adiponectin, adipose tissue triacylglycerol lipase (ATGL), diacylglycerol acyl transferase 1 (DGAT1), glycerol-3-phosphate dehydrogenase 1 (GPD1), and lipoprotein lipase (LPL), but negatively with CD36 and fatty acid-binding protein 4 (FABP4). In preadipocyte culture, ErbB1, but not ErbB2, inhibition was associated with a reduction in the expression of all the above-mentioned genes.

Conclusions: These findings demonstrate a key role for ErbB1 in adipogenesis and suggest that lower ErbB1 protein abundance may lead to adipose tissue dysfunction.

This article appears in The Journal of Clinical Endocrinology \& Metabolism, 10.1210/jc.2011-1033

Genomic Imprinting Effects on Cognitive and Social Abilities in Prepubertal Girls with Turner Syndrome

Jean-François Lepage, David S. Hong, Joachim Hallmayer, and Allan L. Reiss 
Context: Recent evidence suggests that the cognitive and social manifestations associated with Turner syndrome (TS) might be influenced by epigenetic factors in the form of genomic imprinting. However, due to small and heterogeneous samples, inconsistent results have emerged from these studies.

Objective: The objective of this prospective study was to establish the impact of genomic imprinting on neurocognitive abilities and social functioning in young girls with TS.

Design, Setting, and Participants: An extensive battery of neuropsychological assessments was administered to 65 children with TS who had never been exposed to estrogen treatment, 24 of whom had an X-chromosome from paternal origin $\left(\mathrm{X}^{\mathrm{pat}}\right)$ and 41 from maternal origin $\left(\mathrm{X}^{\mathrm{mat}}\right)$.

Main Outcome Measures: The Wechsler scales of intelligence, the Motor-Free Visual Spatial test-3, the Wide Range Assessment of Visual Motor Ability, and the attention/executive domain of the NEPSY were used to assess cognitive abilities. Social functioning was assessed with the Social Responsiveness Scale and the Behavior Assessment System for Children-2.

Results: Results showed that although individuals with $\mathrm{X}^{\text {pat }}$ obtained lower scores than their counterparts with $\mathrm{X}^{\text {mat }}$ on most cognitive and social measures, only the Perceptual Reasoning Index of the intelligence scale yielded significant differences after correction for multiple comparisons.

Conclusion: Overall, these results suggest that although some aspects of the neuropsychological profile of TS may be influenced by epigenetic factors, the sociocognitive phenotype associated with the disorder is not modulated by genomic imprinting.

This article appears in The Journal of Clinical Endocrinology \& Metabolism, 10.1210/jc.2011-2916

\section{Do Phthalates Affect Steroidogenesis by the Human Fetal Testis? Exposure of Human Fetal Testis Xenografts to Di-n-Butyl Phthalate}

R. T. Mitchell, A. J. Childs, R. A. Anderson, S. van den Driesche, P. T. K. Saunders, C. McKinnell, W. H. B. Wallace, C. J. H. Kelnar, and R. M. Sharpe

Context: Phthalates are ubiquitous environmental chemicals. Fetal exposure to certain phthalates [e.g. di-nbutyl phthalate (DBP)] causes masculinization disorders in rats, raising concern for similar effects in humans. We investigated whether DBP exposure impairs steroidogenesis by the human fetal testis.

Objective: The aim of the study was to determine effects of DBP exposure on testosterone production by normally growing human fetal testis xenografts.

Design: Human fetal testes $(14-20$ wk gestation; $n=$ 12) were xenografted into castrate male nude mice that were treated for 4-21 d with vehicle, or $500 \mathrm{mg} / \mathrm{kg} \cdot \mathrm{d}$
DBP, or monobutyl phthalate (active metabolite of DBP); all mice were treated with human chorionic gonadotropin to mimic normal human pregnancy. Rat fetal testis xenografts were exposed for $4 \mathrm{~d}$ to DBP as a positive control.

Main Outcome Measures: Testosterone production was assessed by measuring host serum testosterone and seminal vesicle (SV) weights at termination, plus testis gene expression (rats).

Results: Human fetal testis xenografts showed similar survival $(\sim 80 \%)$ and total graft weight $(8.6$ vs. $10.1 \mathrm{mg})$ in vehicle and DBP-exposed hosts, respectively. Serum testosterone ( 0.56 vs. $0.64 \mathrm{ng} / \mathrm{ml} ; P>0.05)$ and SV weight (67.2 vs. $81.9 \mathrm{mg} ; P>0.05)$ also did not differ. Exposure to monobutyl phthalate gave similar results. In contrast, exposure of rat fetal xenografts to DBP significantly reduced SV weight and testis Cyp11a1/StAR mRNA expression and lowered testosterone levels, confirming that DBP exposure can inhibit steroidogenesis in xenografts, further validating the negative findings on testosterone production in the human.

Conclusions: Exposure of human fetal testes to DBP is unlikely to impair testosterone production as it does in rats. This has important safety and regulatory implications.

This article appears in The Journal of Clinical Endocrinology \& Metabolism, 10.1210/jc.2011-2411

\section{Human Melatonin and Alerting Response to Blue- Enriched Light Depend on a Polymorphism in the Clock Gene PER3}

Sarah L. Chellappa, Antoine U. Viola, Christina Schmidt, Valérie Bachmann, Virginie Gabel, Micheline Maire, Carolin F. Reichert, Amandine Valomon, Thomas Götz, Hans-Peter Landolt, and Christian Cajochen

Context: Light exposure, particularly at the shortwavelength range, triggers several nonvisual responses in humans. However, the extent to which the melatoninsuppressing and alerting effect of light differs among individuals remains unknown.

Objective: Here we investigated whether blue-enriched polychromatic light impacts differentially on melatonin and subjective and objective alertness in healthy participants genotyped for the PERIOD3 (PER3) variable-number, tandem-repeat polymorphism.

Design, Setting, and Participants: Eighteen healthy young men homozygous for the PER3 polymorphism $\left(P E R 3^{5 / 5}\right.$ and $P E R 3^{4 / 4}$ ) underwent a balanced crossover design during the winter season, with light exposure to compact fluorescent lamps of 40 lux at $6500 \mathrm{~K}$ and at $2500 \mathrm{~K}$ during2hinthe evening.

Results: In comparison with the light at $2500 \mathrm{~K}$, blueenriched light at $6500 \mathrm{~K}$ induced a significant suppression of the evening rise in endogenous melatonin levels in $P E R 3^{5 / 5}$ individuals but not in $P E R 3^{4 / 4}$ volunteers. 
Likewise, $P E R 3^{5 / 5}$ individuals exhibited a more pronounced alerting response to light at $6500 \mathrm{~K}$ than $P E R 3^{4 / 4}$ volunteers. Waking electroencephalographic activity in the theta range (5-7 Hz), a putative correlate of sleepiness, was drastically attenuated during light exposure at $6500 \mathrm{~K}$ in $P E R 3^{5 / 5}$ individuals as compared with $P E R 3^{4 / 4}$.

Conclusions: We provide the first evidence that humans homozygous for the $P E R 3^{5 / 5}$ allele are particularly sensitive to blue-enriched light, as indexed by the suppression of endogenous melatonin and waking theta activity. Light sensitivity in humans may be modulated by a clock gene polymorphism implicated in sleep-wake regulation.

This article appears in The Journal of Clinical Endocrinology \& Metabolism, published December 21, 2011, 10.1210/ jc.2011-2391

\section{Maternal Serum Fatty Acid Binding Protein 4 (FABP4) and the Development of Preeclampsia}

Christina M. Scifres, Janet M. Catov, and Hyagriv Simhan

Context: Serum fatty acid binding protein 4 (FABP4) is associated with components of the metabolic syndrome in nonpregnant individuals, including dyslipidemia and insulin resistance. Preeclampsia shares many features with the metabolic syndrome, but the relationship between early pregnancy serum FABP4 levels and the development of preeclampsia is unknown.

Objective: The aim of the study was to test the hypothesis that FABP4 is elevated in women who develop preeclampsia before the onset of disease.

Study Design: This was a nested case-control study within a larger prospective cohort of healthy women with singleton gestations. Cases included 22 women who developed preeclampsia, and a random sample of 72 unmatched controls delivered without preeclampsia was identified. Maternal serum FABP4 was measured at less than 13 wk gestation and 24-28 wk gestation, which was before the onset of preeclampsia in all patients.

Main Outcome Measures: The main outcome measure was preeclampsia (new-onset gestational hypertension and proteinuria for the first time after $20 \mathrm{wk}$ gestation).

Results: Maternal serum FABP4 concentrations were higher in women who ultimately developed preeclampsia both at $8-13 \mathrm{wk}(20.4 \pm 12.3$ vs. $10.1 \pm 4.7 \mathrm{ng} / \mathrm{ml} ; P<0.01)$ and at $24-28 \mathrm{wk}(20.7 \pm 11.7$ vs. $9.9 \pm 4.5 \mathrm{ng} / \mathrm{ml} ; P<0.01)$. After controlling for first trimester body mass index, systolic blood pressure, and nulliparity, FABP4 was associated with the development of preeclampsia (adjusted odds ratio, 1.2; 95\% confidence interval, $1.1-1.3 ; P<0.01)$.

Conclusion: Maternal serum FABP4 levels are elevated before the clinical onset of preeclampsia, and this increase occurs independently of maternal body mass index.
This article appears in The Journal of Clinical Endocrinology \& Metabolism, published December 21, 2011, 10.1210/jc.2011-2276

Increased Activation of the Alternative "Backdoor" Pathway in Patients with 21-Hydroxylase Deficiency: Evidence from Urinary Steroid Hormone Analysis

Clemens Kamrath, Ze'ev Hochberg, Michaela F. Hartmann, Thomas Remer, and Stefan A. Wudy

Background: 17-Hydroxyprogesterone (17-OHP) can be converted to dihydrotestosterone (DHT) via an alternative "backdoor" route that bypasses the conventional intermediates androstenedione and testosterone. In this backdoor pathway, $17 \mathrm{OHP}$ is converted to $5 \alpha$-pregnane$3 \alpha, 17 \alpha$-diol-20-one (pdiol), which is an excellent substrate for the 17,20 lyase activity of CYP17A1 to produce androsterone.

Objective and Hypotheses: The objective of this study was to obtain evidence for the presence of the backdoor pathway in patients with 21-hydroxylase deficiency (21OHD).

Methods: We compared urinary steroid hormone profiles determined by gas chromatography-mass spectrometry of 142 untreated 21-OHD patients (age range, $1 \mathrm{~d}$ to $25.4 \mathrm{yr}$; 51 males) with 138 control subjects. The activity of the backdoor pathway was assessed using the ratios of the urinary concentrations of pdiol to those of the metabolites of the classic $\Delta^{4}$ and $\Delta^{5}$ pathways. In contrast to etiocholanolone, which originates almost exclusively from the classic pathways, androsterone may be derived additionally from the backdoor pathway. Therefore, the androsterone to etiocholanolone ratio can be used as an indicator for the presence of the backdoor pathway.

Results: Untreated 21-OHD subjects showed increased urinary ratios of pdiol to the $\Delta^{4}$ and $\Delta^{5}$ pathway metabolites and a higher androsterone to etiocholanolone ratio.

Conclusions: The elevated ratios of pdiol to the $\Delta^{4}$ and $\Delta^{5}$ pathway metabolites as well as the higher androsterone to etiocholanolone ratio in patients with 21-OHD indicate postnatal activity of the backdoor pathway with maximum activity during early infancy. Our data provide new insights into the pathophysiology of androgen biosynthesis of 21OHD.

This article appears in The Journal of Clinical Endocrinology \& Metabolism, published December 14, 2011, 10.1210/ jc.2011-1997

Succinate Dehydrogenase (SDH) D Subunit (SDHD) Inactivation in a Growth-Hormone-Producing Pituitary Tumor: A New Association for SDH?

Paraskevi Xekouki, Karel Pacak, Madson Almeida, Christopher A. Wassif, Pierre Rustin, Maria Nesterova, Maria de la Luz Sierra, Joey Matro, Evan Ball, Monalisa Azevedo, 
Anelia Horvath, Charalampos Lyssikatos, Martha Quezado, Nicholas Patronas, Barbara Ferrando, Barbara Pasini, Aristides Lytras, George Tolis, and Constantine A. Stratakis

Background: Mutations in the subunits B, C, and D of succinate dehydrogenase (SDH) mitochondrial complex II have been associated with the development of paragangliomas (PGL), gastrointestinal stromal tumors, papillary thyroid and renal carcinoma (SDHB), and testicular seminoma (SDHD).

Aim: Our aim was to examine the possible causative link between $S D H D$ inactivation and somatotropinoma.

Patients and Methods: A 37-yr-old male presented with acromegaly and hypertension. Other family members were found with PGL. Elevated plasma and urinary levels of catecholamines led to the identification of multiple PGL in the proband in the neck, thorax, and abdomen. Adrenalectomy was performed for bilateral pheochromocytomas (PHEO). A GH-secreting macroadenoma was also found and partially removed via trans-sphenoidal surgery (TTS). Genetic analysis revealed a novel SDHD mutation (c.298_301delACTC), leading to a frame shift and a premature stop codon at position 133 of the protein. Loss of heterozygosity for the $S D H D$ genetic locus was shown in the GH-secreting adenoma. Down-regulation of SDHD protein in the GH-secreting adenoma by immunoblotting and immunohistochemistry was found. A literature search identified other cases of multiple PGL/PHEO in association with pituitary tumors.

Conclusion: We describe the first kindred with a germline $S D H D$ pathogenic mutation, inherited PGL, and acromegaly due to a GH-producing pituitary adenoma. SDHD loss of heterozygosity, down-regulation of protein in the GHsecreting adenoma, and decreased SDH enzymatic activity supports $S D H D$ 's involvement in the pituitary tumor formation in this patient. Older cases of multiple PGL and PHEO and pituitary tumors in the literature support a possible association between SDH defects and pituitary tumorigenesis.

This article appears in The Journal of Clinical Endocrinology \& Metabolism, published December 14, 2011, 10.1210/ jc. 2011-1179

Krüppel-Like Factor 9 and Progesterone Receptor Coregulation of Decidualizing Endometrial Stromal Cells: Implications for the Pathogenesis of Endometriosis

John Mark P. Pabona, Frank A. Simmen, Mikhail A. Nikiforov, DaZhong Zhuang, Kartik Shankar, Michael C. Velarde, Zara Zelenko, Linda C. Giudice, and Rosalia C.M. Simmen

Context: Endometriosis is characterized by progesterone resistance and associated with infertility. Krüppel-like factor 9 (KLF9) is a progesterone receptor (PGR)-interacting protein, and mice null for Klf9 are subfertile. Whether loss of KLF9 expression contributes to progesterone resistance of eutopic endometrium of women with endometriosis is unknown.

Objective: The aims were to investigate 1) KLF9 expression in eutopic endometrium of women with and without endometriosis, 2) effects of attenuated KLF9 expression on WNT-signaling component expression and on WNT inhibitor Dickkopf-1 promoter activity in human endometrial stromal cells (HESC), and 3) PGR and KLF9 coregulation of the stromal transcriptome network.

Methods: Transcript levels of KLF9, PGR, and WNT signaling components were measured in eutopic endometrium of women with and without endometriosis. Transcript and protein levels of Wnt signaling components in HESC transfected with KLF9 and/or PGR small interfering RNA were analyzed by quantitative RT-PCR and Western blot. KLF9 and PGR coregulation of Dickkopf-1 promoter activity was evaluated using human Dickkopf-1-luciferase promoter/reporter constructs and by chromatin immunoprecipitation. KLF9 and PGR signaling networks were analyzed by gene expression array profiling.

Results: Eutopic endometrium from women with endometriosis had reduced expression of KLF9 mRNA together with those of $P G R-B, W N T 4, W N T 2$, and DKK1. KLF9 and PGR were recruited to the $D K K 1$ promoter and modified each other's transactivity. In HESC, KLF9 and PGR coregulated components of the Wnt, cytokine, and IGF gene networks that are implicated in endometriosis and infertility.

Conclusion: Loss of KLF9 coregulation of endometrial stromal PGR-responsive gene networks may underlie progesterone resistance in endometriosis.

This article appears in The Journal of Clinical Endocrinology \& Metabolism, 10.1210/jc.2011-2562

\section{Shorter Androgen Receptor CAG Repeat Lengths Associated with Cryptorchidism Risk among Hispanic White Boys}

Carol Davis-Dao, Chester J. Koh, Brian E. Hardy, Andy Chang, Steve S. Kim, Roger De Filippo, Andrew Hwang, Malcolm C. Pike, Joshua D. Carroll, Gerhard A. Coetzee, David Vandenberg, Kimberly Siegmund, and Victoria K. Cortessis

Context: Cryptorchidism is the most frequent congenital malformation among males, the major established risk factor for testicular germ cell tumors, and a presumed infertility risk factor. Androgens are essential for testicular descent, and functional genetic polymorphisms in the androgen receptor gene $(A R)$ are postulated to influence cryptorchidism risk.

Objective: The aim of the study was to investigate whether the CAG repeat length polymorphism in exon 1 of the $A R$ is associated with cryptorchidism risk.

Design and Setting: We conducted a family-based genotype-risk association study employing the transmission disequilibrium test for genotypic variants transmitted on the 
X-chromosome at a university-affiliated regional children's hospital.

Participants: We studied 127 Hispanic boys with persistent cryptorchidism and comorbidities described in detail and their biological mothers.

Intervention: Genotypes defined by number of $\mathrm{CAG}$ repeats were measured for each member of participating son-mother pairs.

Main Outcome Measure: Associations between CAG tract length genotype and cryptorchidism risk were estimated using matched-pairs logistic regression.

Results: Cryptorchidism risk was significantly associated with shorter $\mathrm{CAG}$ repeats $[\mathrm{CAG} \leq 19$ vs. $\mathrm{CAG} \geq 20$, odds ratio $(\mathrm{OR})=0.44 ; 95 \%$ confidence interval $(\mathrm{CI}), 0.23-0.88]$. This association was restricted to cryptorchidism with accompanying comorbidities, which was primarily hernia $[\mathrm{CAG} \leq 19$ vs. $\mathrm{CAG} \geq 20, \mathrm{OR}=0.35$ (95\% CI, 0.16-0.78)], and was strongest for bilateral cryptorchidism $[\mathrm{CAG} \leq 19$ vs. CAG $\geq$ $20, \mathrm{OR}=0.09(95 \% \mathrm{CI}, 0.010-0.78)]$.

Conclusions: Androgen receptor genotypes encoding moderate functional variation may influence cryptorchidism risk, particularly among boys with bilateral nondescent or congenital hernia, and may explain in part the elevated risk of testicular seminoma experienced by ex-cryptorchid boys. Mechanistic research is warranted to examine both classical and nonclassical mechanisms through which androgens may influence risk of cryptorchidism and related conditions.

This article appears in The Journal of Clinical Endocrinology \& Metabolism, published December 21, 2011, 10.1210/ jc. 2011-2439

Orbit-Infiltrating Mast Cells, Monocytes, and Macrophages Produce PDGF Isoforms that Orchestrate Orbital Fibroblast Activation in Graves' Ophthalmopathy

L. van Steensel, D. Paridaens, M. van Meurs, P. M. van Hagen, W. A. van den Bosch, R. W. A. M. Kuijpers, H. A. Drexhage, H. Hooijkaas, and W. A. Dik
Purpose: Platelet-derived growth factors (PDGF) are regulators of fibroblast activity that may be involved in the pathophysiology of Graves' ophthalmopathy (GO). We unraveled the expression and origin of PDGF family members in GO orbital tissue and investigated the effect of PDGF isoforms on IL-6 and hyaluronan production and proliferation by orbital fibroblasts.

Methods: PDGF-A, PDGF-B, PDGF-C, PDGF-D, PDGF$\mathrm{R} \alpha$, and PDGF-R $\beta$ expression was determined by real-time quantitative, PCR and PDGF-A and PDGF-B protein expression was determined by Western blot in orbital tissues. Orbital tissues were immunohistochemically stained for PDGF-A and PDGF-B expression, together with stainings for $\mathrm{T}$ cells, monocytes, B cells, macrophages, and mast cells. Effects of PDGF-AA, PDGF-AB, and PDGF-BB on orbital fibroblast proliferation and IL- 6 and hyaluronan production were examined. Finally, effects of PDGF-BB-and PDGF-AAneutralizing antibodies on IL- 6 and hyaluronan production in GO whole orbital tissue cultures were tested.

Results: GO orbital tissue showed increased PDGF-A and PDGF-B mRNA and protein levels. Increased numbers of PDGF-A-and PDGF-B-positive monocytes, macrophages, and mast cells were present in GO orbital tissue. PDGF$\mathrm{BB}$ stimulated proliferation and hyaluronan and IL-6 production by orbital fibroblasts the most, followed by PDGF$\mathrm{AB}$ and PDGF-AA. Finally, in particular imatinib mesylate and PDGF-BB-neutralizing antibodies reduced IL-6 and hyaluronan production by whole orbital tissue cultures from GO patients.

Conclusions: In GO, mast cells, monocytes, and macrophages may activate orbital fibroblasts via secretion of especially PDGF-AB and PDGF-BB. Preclinical studies with whole orbital tissue cultures show that blocking PDGF-B chain containing isoforms can be a promising treatment for GO.

This article appears in The Journal of Clinical Endocrinology \& Metabolism, 10.1210/jc.2011-2697 